\title{
Der Einfluß des Prostomiums auf die Entwicklung der Geschlechtsprodukte des Polychaeten Anaitides mucosa (Phyllodocidae)
}

\author{
A. Röhrkasten \\ Fachbereich Biologie der Universität Oldenburg; \\ Ammerländer Heerstr. 67-99, D-2900 Oldenburg, Bundesrepublik Deutschland
}

\begin{abstract}
The influence of the prostomium on the sexual maturity of the polychaete Anaitides mucosa (Phyllodocidae). Spermatogenesis and especially oogenesis have been investigated in decerebrate and intact specimens of Anaitides mucosa. The results indicate, in contrast to similar experiments on the related species Eulalia viridis, that decerebration causes an accelerated, but nevertheless qualitative normal growth of coelomic oocytes. The implantation of whole prostomia into the coelom of decerebrate females has no counteractive effects. It is likely that the prostomium exerts its influence via the ventral nerve cord.
\end{abstract}

\section{EINLEITUNG}

Innerhalb der Klasse der Polychaeten besteht eine große Vielfalt hinsichtlich der Beteiligung endokriner Mechanismen an der geschlechtlichen Entwicklung und der Fortpflanzung. Mit Ausnahme der Sylliden konnte in allen Fällen das Prostomium als Bildungsstätte der experimentell nachgewiesenen Hormone identifiziert werden. Da hierbei jedoch die Anzahl der untersuchten Arten, die nicht zu den beiden relativ umfassend behandelten Familien der Nereiden und der Sylliden gehören, noch sehr spärlich ist, ist die Aufstellung einheitlicher Prinzipien der hormonalen Kontrolle noch mit vielen Fragezeichen verbunden. Zusammenfassende Darstellungen hierzu geben Golding (1974), Hauenschild (1974, 1975), Olive (1979) sowie Olive \& Clark (1978).

Innerhalb der Familie der Phyllodocidae ist bisher lediglich die Art Eulalia viridis Gegenstand umfangreicher Untersuchungen gewesen (Olive, 1975, 1976; Olive \& Moore, 1975). Die Ergebnisse zeigen die Zugehörigkeit dieser Art zur Gruppe der polytelischen Polychaeten mit gonadotrophen Hormonen. Die Amputation des Prostomiums führt somit zur Degeneration der noch unreifen Oocyten. Dieser Effekt läßt sich durch die gleichzeitige Implantation von zwei vollständigen Prostomien in das Coelom des dekapitierten Weibchens wieder aufheben.

Die vorliegende Arbeit über Anaitides mucosa (Abb. 1, 2) gibt Aufschluß darüber, inwieweit auch in der Familie der Phyllodocidae Übereinstimmungen hinsichtlich der hormonalen Kontrolle der geschlechtlichen Entwicklung auftreten. 


\section{MATERIAL UND METHODEN}

\section{Untersuchungsobjekt}

Untersuchungsobjekt ist die arktisch-amphiboreal-mediterrane Phyllodocide Anaitides mucosa. Die Versuchstiere wurden während eines Aufenthaltes im meeresbiologischen Institut der Universität Lille in Wimereux Ende August 1980 gesammelt.

Die Unterscheidung der Geschlechter ist nur anhand der Geschlechtsprodukte möglich. In Übereinstimmung mit anderen Phyllodociden (Gravier, 1896) fehlen auch bei $A$. mucosa engumgrenzte Gonaden. Statt dessen entwickeln sich die Gametocyten aus frei in der Coelomflüssigkeit flottierenden Zellpaketen, deren Ursprung umstritten ist. Die hier besonders interessierende Vitellogenese durchlaufen die Oocyten als Einzelzellen in der Coelomflüssigkeit. Die Sexualreifung findet zwischen Anfang November und Mitte Februar statt.

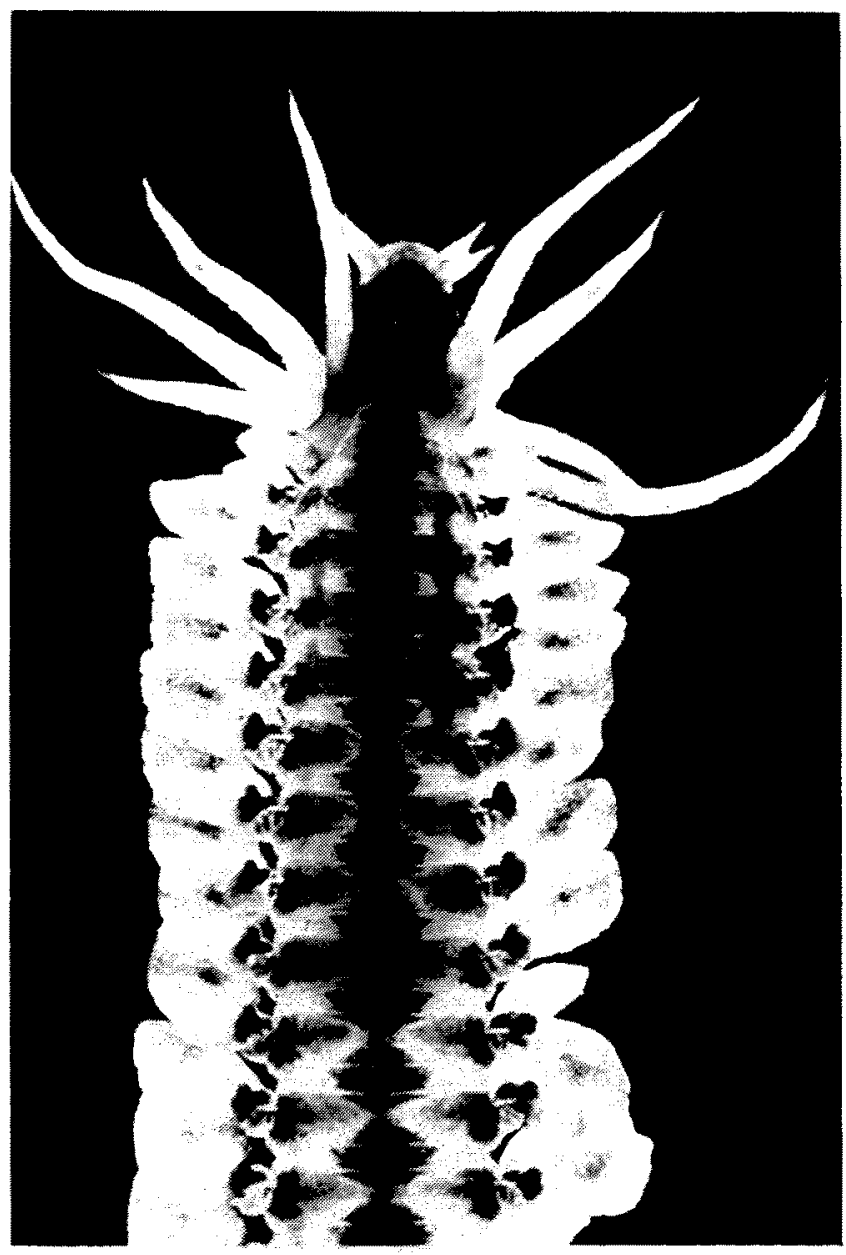

Abb. 1. Anaitides mucosa (Vorderende von dorsal) 


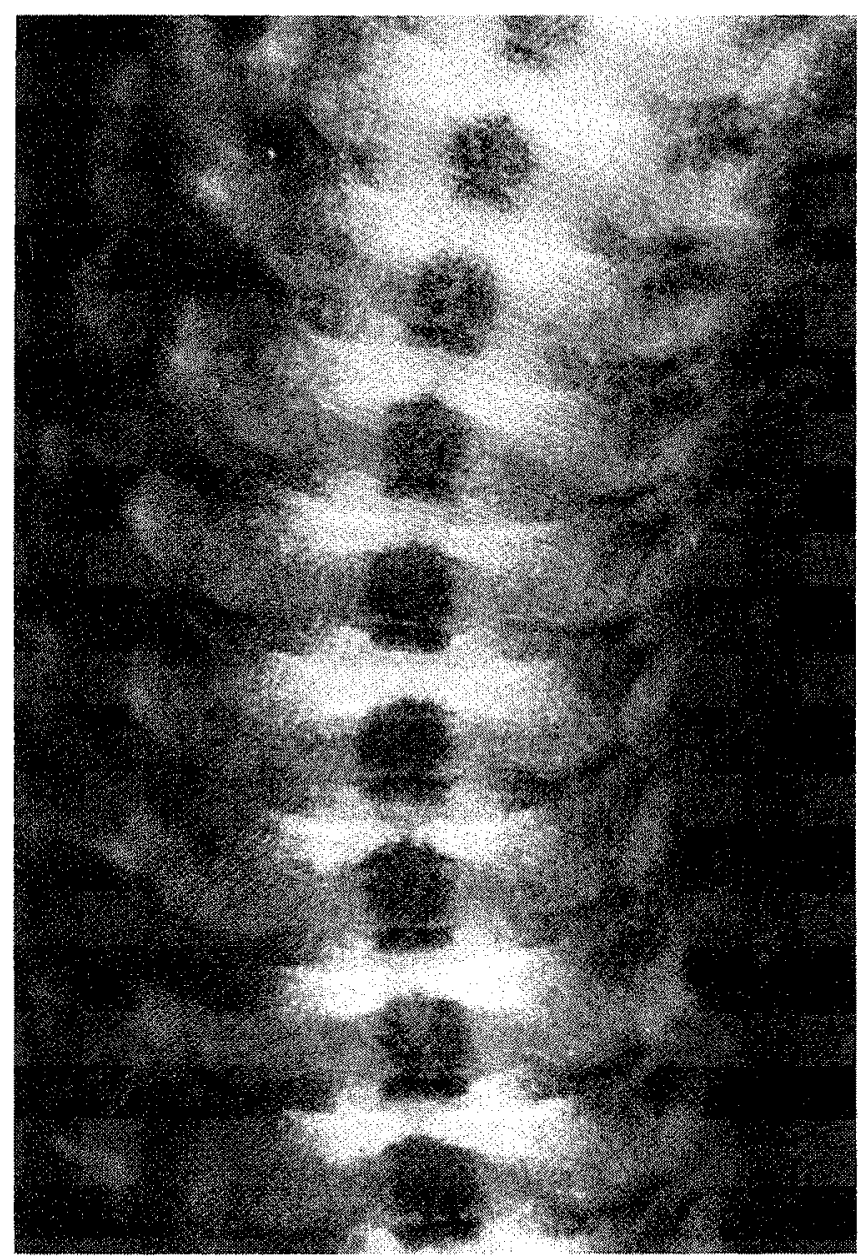

Abb. 2. Mehrere Segmente aus der mittleren Körperregion eines Anaitides- $q$ mit fast reifen Eizellen

Ausführlichere Angaben zur Fortpflanzungsbiologie von A. mucosa liefern Sach (1975) und Cazaux (1969).

\section{Kulturmethode}

Die Kultur der Würmer erfolgte in belüfteten Polystyrol-Behältern mit $1000 \mathrm{ml}$ Kulturmedium für etwa 90 Tiere. Der Boden der Gefäße war mit einer dünnen Kiesschicht überzogen, um den Tieren die Möglichkeit zum Verkriechen zu geben. Für die Unterbringung der Würmer während der Experimente dienten entweder Petrischalen ( $\varnothing 5 \mathrm{~cm}$; Höhe des Wasserstandes: $0,5 \mathrm{~cm}$ ), in denen die Tiere einzeln gehalten wurden, oder ebenfalls Polystyrol-Behälter (150 ml Kulturmedium), die mit maximal 15 durch Parapodien-Entfernung individuell markierten Versuchstieren besetzt wurden. Als Kul- 
turmedium wurde künstliches, vor Gebrauch zusätzlich bei $80^{\circ} \mathrm{C}$ sterilisiertes Seewasser (Tropic Marin Neu) verwendet. Die Hälterung der Tiere erfolgte sowohl vor als auch während des eigentlichen Versuchszeitraums hinsichtlich Temperatur und Tageslänge unter den der Jahreszeit entsprechenden Bedingungen.

\section{Operationstechnik}

Alle Untersuchungen und operativen Eingriffe wurden an betäubten Würmern vorgenommen. Nach 5-10minütiger Narkose in einer dem Kulturmedium isotonischen $\mathrm{MgCl}_{2}$-Lösung wurden die Operatioṇen auf einem Objektträger unter einem Binokular bei $20 f a c h e r$ Vergrößerung durchgeführt.

Messung der Oocytengröße: Zu diesem Zweck wurden den Versuchstieren in der mittleren Körperregion mit einer feinen Schere einseitig zwei oder drei Parapodien entfernt und die Oocyten der betroffenen Segmente vorsichtig aus dem Coelom herausgedrückt. Die eigentliche Messung der Oocytengröße erfolgte dann unter einem Mikroskop bei 400facher Vergrößerung. Es wurden stets nur intakte, in der Aufsicht annähernd kreisrunde Oocyten gemessen; bei ovalen Eizellen wurde der Mittelwert zwischen größtem und kleinstem Durchmesser bestimmt (Hauenschild, 1966).

Zur Klassifizierung der Anaitides- $q$ h hinsichtlich der Oocyten-Reifung diente das folgende, empirisch erstellte System (in Klammern: Anzahl der den einzelnen Klassen experimentell zugewiesenen Einzelwerte). Der für jedes Versuchstier gewonnene Mittelwert basiert, sofern keine anderslautenden Hinweise gegeben werden, auf jeweils 40 ausgemessenen Eizellen:

Klass e 1 (90): Alle Oocyten sind kleiner als $40 \mu \mathrm{m}$ im Durchmeser; im Mittel bleiben die Werte unter $36,0 \mu \mathrm{m}$.

Klasse 2 (226): Der überwiegende Anteil der Oocyten liegt zwischen 40 und $60 \mu \mathrm{m}$ im $\varnothing_{i}$ der Mittelwert bewegt sich zwischen 36,1 und 55,0 $\mu \mathrm{m}$.

$\mathrm{K} l$ as s e 3 (257): Die Mehrheit der Oocyten liegt zwischen 60 und $80 \mu \mathrm{m}$ im $\varnothing$, der Mittelwert zwischen 55,1 und 76,0 $\mu \mathrm{m}$.

Klasse 4 (310): Der überwiegende Anteil der Oocyten liegt zwischen 80 und $100 \mu \mathrm{m}$ im $\varnothing$, der Mittelwert zwischen 76,1 und 99,0 $\mu \mathrm{m}$.

Klasse 5 (132): Die meisten Oocyten sind größer als $100 \mu \mathrm{m}$ im $\varnothing_{\text {; im Mittel }}$ liegen die Werte über $99,1 \mu \mathrm{m}$.

Prostomium-Amputation: Die Amputation des Prostomiums erfolgte durch einen hinter den Augen querverlaufenden Schnitt mit einem Rasierklingensplitter, wobei Peristomium und Tentakelzirren unversehrt blieben.

Prostomium-Implantation: Das zur Implantation bestimmte Prostomium wurde durch eine nach einseitiger Entfernung von 1-2 Parapodien entstandene Öffnung innerhalb der ersten zehn Segmente in das Coelom des Empfängers mit einer Präpariernadel hineingesteckt und dann ventral vom Darm vorsichtig in den Bereich der Segmente 25 bis 40 geschoben. Dies ist auf Grund der unvollständigen Ausbildung der Dissepimente bei A. mucosa möglich.

Um die Folgen der Amputation des Prostomiums auf den Ablauf der Oogenese zu beobachten, wurden in der Zeit vom 20. Oktober 1980 bis zum 15. Februar 1981 insgesamt vier Versuchsreihen im Abstand von jeweils 3-5 Wochen begonnen. Die 
Kontrolluntersuchungen erfolgten nach jeweils 31 Versuchstagen, solange bis die Mehrzahl der Tiere mindestens einer der zu vergleichenden Versuchsgruppen ihre OocytenEntwicklung nahezu vollendet hatte. Da prostomiumslose Tiere unfähig zur Nahrungsaufnahme sind, wurden alle Versuchstiere während des gesamten Versuchszeitraums nicht gefüttert. Dies war deshalb zulässig, weil sich in Vorversuchen gezeigt hatte, daß ein Anaitides- + , dessen mittlerer Oocyten-Durchmesser bei Versuchsbeginn bei $50 \mu \mathrm{m}$ lag, durchaus imstande war, eine 8monatige Hungerperiode unbeschadet zu überstehen.

\section{ERGEBNISSE}

\section{Entwicklung der Oocyten nach Amputation des Prostomiums}

Versuchsreihe 1 (20. Oktober 80 bis 22. Januar 81): Den insgesamt 50 dekapitierten Versuchstieren wurden eine gleich große Kontrollgruppe intakter Hungertiere gegenübergestellt (mittlere Segmentanzahl beider Versuchsgruppen bei Versuchsbeginn: 198,8 bzw. 187,0). Die Vergrößerung der Versuchsgruppen auf je 50 Tiere gegenüber der sonst üblichen Anzahl von maximal 20 Würmern wurde dadurch notwendig, daß anfänglich keinerlei Geschlechtsprodukte, weder äußerlich noch durch Entnahme einer geringen Menge Coelomflüssigkeit, zu erkennen waren. Diese Maßnahme sollte somit sicherstellen, daß sich unter den Versuchstieren genügend weibliche Tiere befanden. Die entsprechenden Ergebnisse sind in Abb. 3 dargestellt. Normalerweise wurden dazu je Versuchstier 40 Oocyten ausgemessen; lediglich bei der ersten Kontrolluntersuchung mußte, um eine größere Verletzung der Tiere bei der Gewinnung der vielfach nur spärlich vorhandenen Eizellen zu vermeiden, eine Beschränkung auf je $30 \mathrm{bzw}$. in zwei Fällen sogar auf je 15 Oocyten erfolgen.

Von den 50 intakten Hungertieren erwiesen sich nach den ersten 31 Versuchstagen 25 als $q Q$ und 15 als $\delta^{\star}$, während 10 Tiere auch weiterhin keine nachweisbaren Geschlechtsprodukte besaßen. Unter den 48 überlebenden prostomiumslosen Tieren waren 26 weiblichen und 22 männlichen Geschlechts. Nur die eindeutig als Weibchen identifizierten Tiere blieben für weitere 62 Tage im Experiment.

Der durch die Amputation des Prostomiums hervorgerufene Effekt zeigt sich nicht nur anhand der in Abb. 3 wiedergegebenen Beschleunigung der Oocytenentwicklung, sondern auch durch seinen gleichartigen Einfluß auf die Spermatogenese. Während alle 15 intakten $\delta \partial$ lediglich Spermatogonien in ihrem Coelom aufwiesen, fanden sich unter den 22 prostomiumlosen ơ nur 4 Tiere mit Spermatogonien, 8 Tiere mit allen der im Rahmen der Entwicklung der männlichen Geschlechtsprodukte durchlaufenen Stadien und 10 Tiere, bei denen die beweglichen Spermien bereits deutlich dominierten.

Von den 26 prostomiumlosen $q q$ (mittlerer Oocyten- $\varnothing$ nach 31 Versuchstagen $\overline{\mathrm{x}}_{31}=52,9 \mu \mathrm{m}$ ) zeigen insgesamt 12 Tiere (ca. $46 \%$ ) einen zum Teil beträchtlichen Vorsprung in bezug auf ihre Oocyten-Größe gegenüber dem am weitesten in seiner Entwicklung fortgeschrittenen $\rho$ unter den 25 unversehrten Kontrolltieren $\left(\bar{x}_{31}=36,7 \mu \mathrm{m}\right)$. Dieser Unterschied wächst nach 62 Versuchstagen sogar noch auf rund $62 \%$ (16 Tiere) an. Der mittlere Oocyten- $\varnothing$ der intakten Versuchstiere liegt dann bei $48,1 \mu \mathrm{m}$, derjenige der dekapitierten Tiere bei $78,3 \mu \mathrm{m}$. Dies entspricht nahezu einer Verdoppelung der Differenz der beiden im Rahmen der ersten Kontrolluntersuchung erhaltenen Werte. 
Nach 93 Versuchstagen zeigt dieser Abstand eine leicht rückläufige Tendenz, nicht zuletzt durch das einem weiteren Wachstum Einhalt gebietende Erreichen der maximalen Oocyten-Größe bei einigen der prostomiumlosen Tiere verursacht.

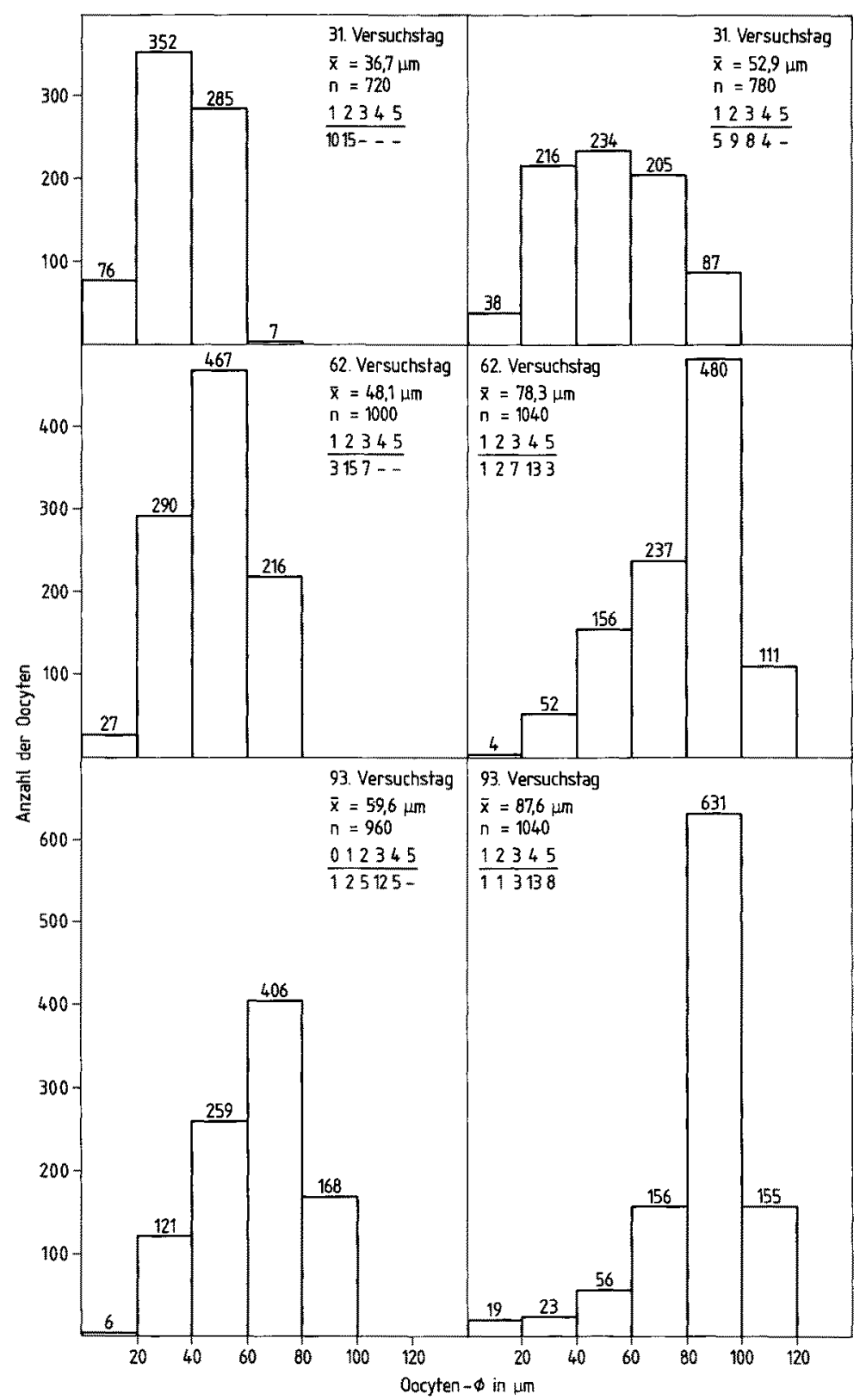

Abb. 3. Verteilung der Oocytengröße von Hungertieren (links) und dekapitierten Tieren (rechts) im Rahmen der Versuchsreihe $1 ; \overline{\mathbf{x}}=$ mittlerer Oocyten- $\phi_{;} \mathfrak{n}=$ Anzahl der ausgemessenen Oocyten 
Die Uberprüfung der durch die Dekapitienung herbeigeführten Beschleunigung der Oocyten-Entwicklung gegenüber derjenigen bei intakten Hungertieren auf einen qualitativ normalen Verlauf hin erfolgte am 8. Februar 81 im Rahmen einer künstlichen Befruchtung. Die reifen Eier von vier prostomiumlosen $q \circ$ wurden mit dem Sperma eines einer gewöhnlichen Massenkultur entnommenen $\delta$ in Kontakt gebracht. In allen vier Fällen zeigten sich 3 bis 4 Tage später frei schwimmende Trochophorae.

Versuchsreihe 2 (12. November 80 bis 15. Februar 81): Bei Beginn dieser zweiten Versuchsreihe war bereits eine Unterscheidung der Geschlechter möglich; die Mehrzahl der ausschließlich weiblichen Versuchstiere gehörte hinsichtlich des Reifegrads der Oocyten der Klasse 1 an. Die mittlere Segmentanzahl der beiden Versuchsgruppen lag bei 214,6 bzw. 224,1.

Nach 31 Versuchstagen zeigt sich bereits wieder ein signifikanter Unterschied zwischen den beiden Versuchsgruppen (Abb. 4). Die unversehrten Hungertiere erreichen innerhalb dieser Zeitspanne nur rund $58 \%$ des Oocyten-Wachstums der dekapitierten Versuchstiere; während die Mehrzahl der Kontrolltiere (13 Tiere $=65 \%$ ) zu diesem Zeitpunkt noch der Klasse 3 angehört, befinden sich die meisten prostomiumlosen Tiere $(13$ Tiere $=65 \%)$ bereits in Klasse 4 . Nach 62 Versuchstagen ist dieser Abstand in etwa gleich geblieben; es ist lediglich eine Verschiebung des Maximums innerhalb der beiden Versuchsgruppen um je eine Klasse erfolgt. Die relative Ännäherung nach 93 Versuchstagen ist wiederum dem Umstand zuzuschreiben, daß der überwiegende Anteil der prostomiumlosen Versuchstiere seine Oocyten-Entwicklung bereits früher vollendet hat und somit ein weiterer Zuwachs des mittleren Oocyten- $\varnothing$ innerhalb der gesamten. Versuchsgruppe nur noch in beschränktem Umfange möglich ist.

Versuchsreihe 3 (30. November 80 bis 2. Februar 81): Hinsichtlich der Durchführung galten im wesentlichen die diesbezüglichen Anmerkungen der bisherigen beiden Versuchsreihen. Zusätzliche Untersuchungen nach 10, 15 und 47 Versuchstagen (Abb. 6) jeweils der Hälfte der beiden aus je 20 Würmern bestehenden Versuchsgruppen (mittlere Segmentanzahl bei Versuchsbeginn: $207,3 \mathrm{bzw} .213,8$ ) sollten neben den üblichen Untersuchungen näheren Aufschluß über die zeitliche Abfolge der durch die Prostomium-Entfernung hervorgerufenen Vorgänge geben. Da die Mehrzahl der Versuchstiere bei Versuchsbeginn bereits bezüglich des Reifegrads der Oocyten der Klasse 2 angehörte, war eine Beschränkung der Versuchsdauer auf 62 Versuchstage möglich.

Die in Abb. 5 dargestellten Ergebnisse entsprechen grundsätzlich denen der vorangegangenen Versuchsreihen, wenn auch der durch die Dekapitierung hervorgerufene Wachstumsschub der Oocyten nach 31 Versuchstagen deutlich geringer als erwartet ausfällt. Hierin zeigen sich die Grenzen und die zweifellos auch vorhandene Ungenauigkeit, die dieser Untersuchungsmethode, basierend auf dem Mittelwert einer jeweils nur 20 Tiere umfassenden Versuchsgruppe, zwangsweise innewohnen müssen. Da drei der dekapitierten Versuchstiere insgesamt ein geringeres Oocyten-Wachstum als jedes der intakten Kontrolltiere zeigen, führt dies sogleich zu einer beträchtlichen Verzerrung der Ergebnisse bei ausschließlicher Betrachtung der Mittelwerte beider Versuchsgruppen. In diesem Sinne müssen auch die Ergebnisse der Abb. 6 gesehen werden, die trotz ihrer vorhandenen Streuung durch ihre zeitliche Abfolge Anlaß zu der Vermutung geben, daß durch die Dekapitierung ein von seiten des Prostomiums ausge- 


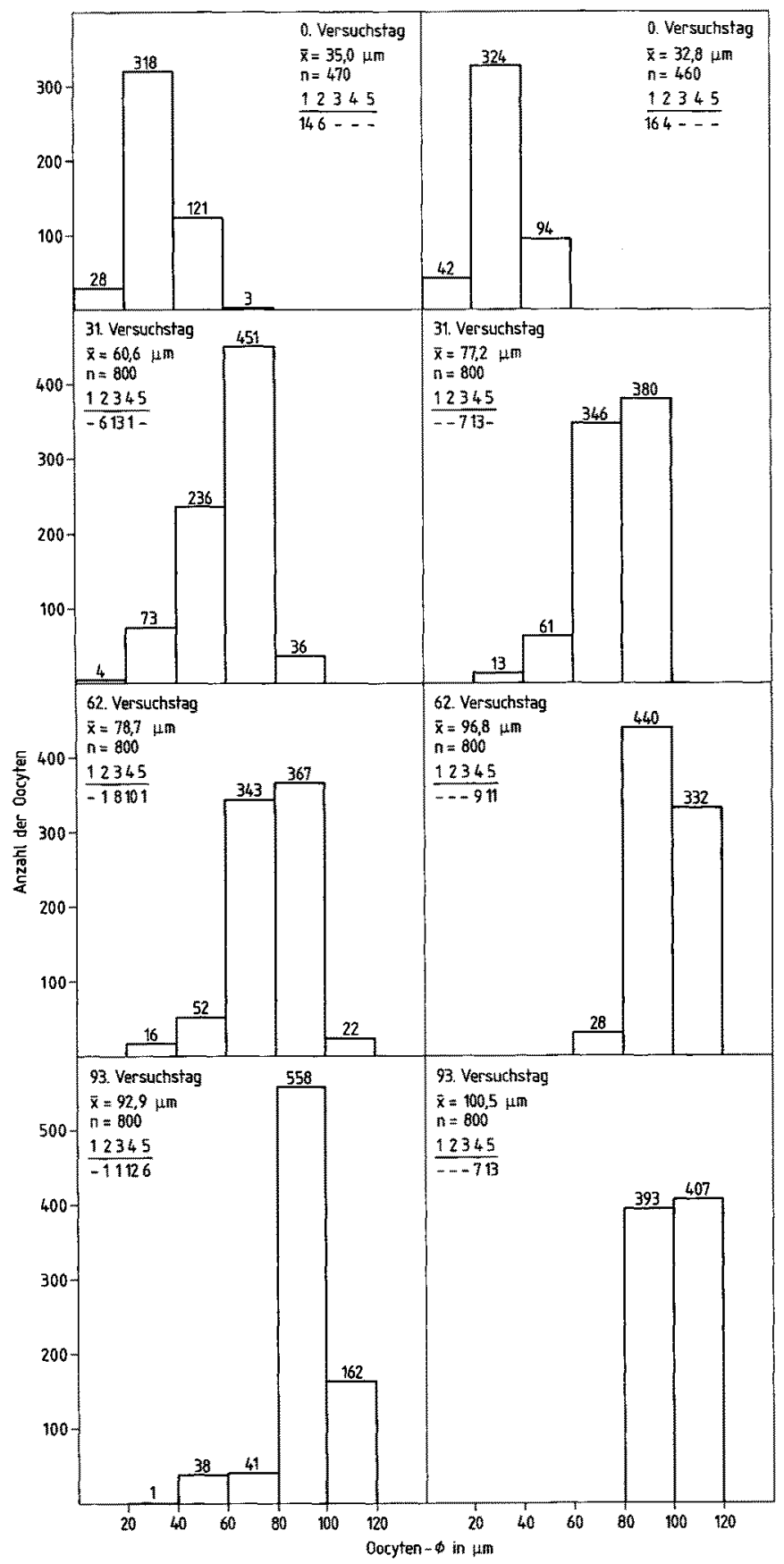

Abb. 4. Verteilung der Oocytengröße von Hungertieren (links) und dekapitierten Tieren (rechts) im Rahmen der Versuchsreihe 2 
hender, die Oocyten-Entwicklung verlangsamender, möglicherweise im Hinblick auf die eigentliche Fortpflanzungszeit ausrichtender Einfluß schlagartig aufgehoben wird, was insbesondere in der zweiten und dritten postoperativen Versuchswoche ein außerordentlich beschleunigtes Oocyten-Wachstum zur Folge hat.

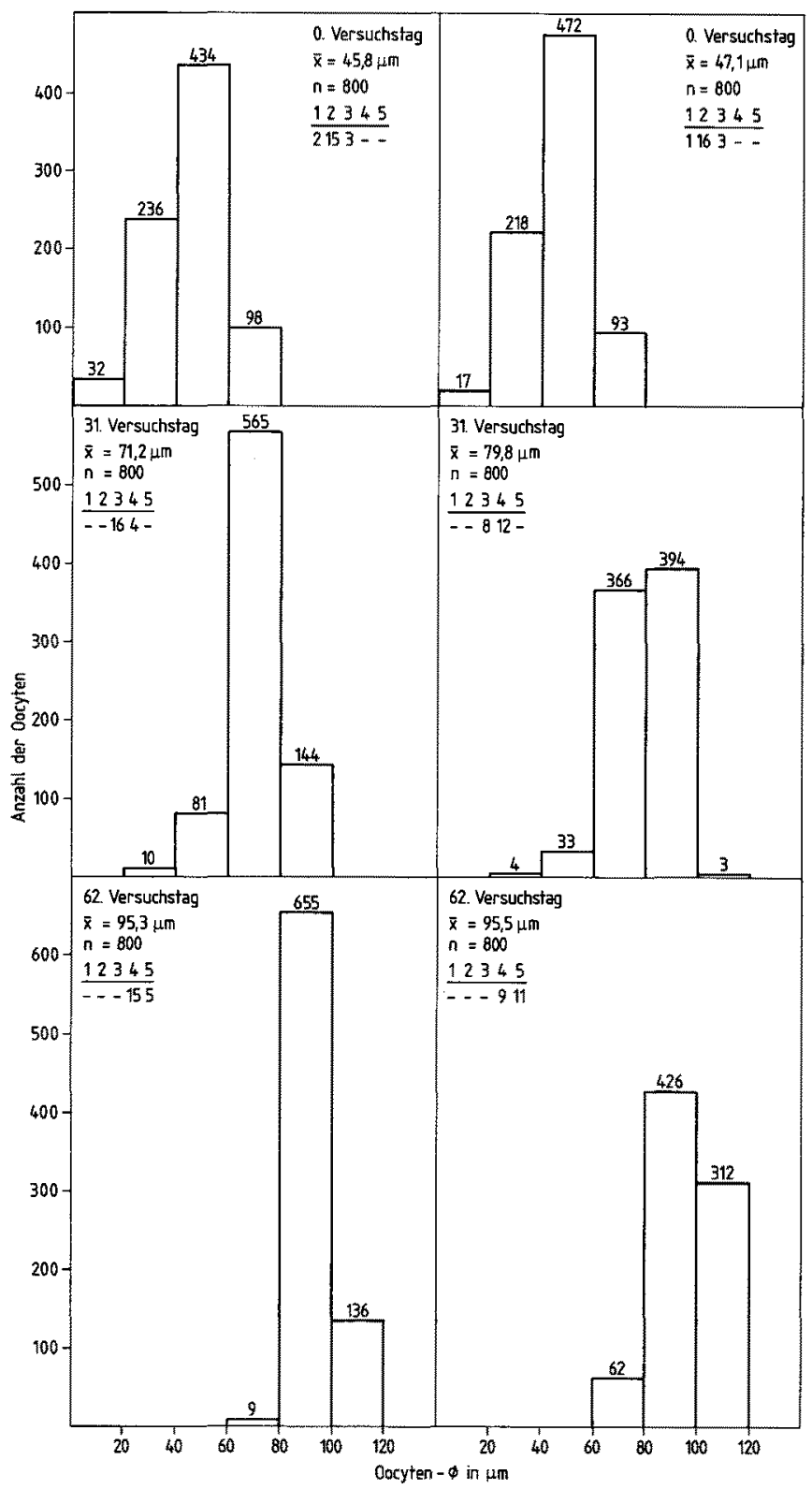

Abb. 5. Verteilung der Oocytengröße von Hungertieren (links) und dekapitierten Tieren (rechts) im Rahmen der Versuchsreihe 3 


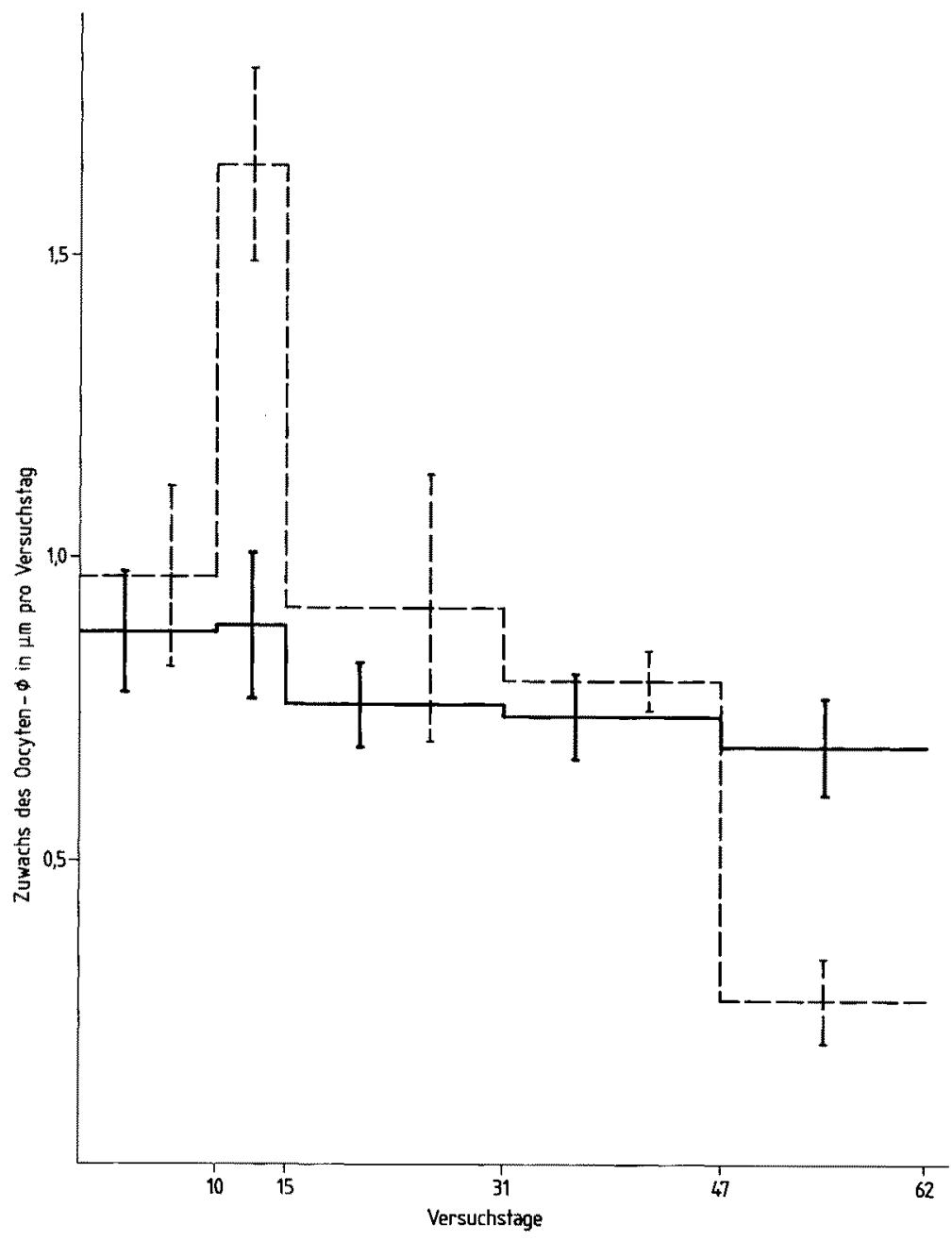

Abb. 6. Mittlerer Zuwachs des Oocyten- $\varnothing$ und mittlerer Fehler des Mittelwertes $(n=10)$ von Hungertieren (_ - ) und Tieren nach Amputation des Prostomiums (- - - ) für die angegebenen Intervalle im Rahmen der Versuchsreihe 2

Versuchsreihe 4 (5. Januar 81 bis 6. Februar 81): Die letzte der im Rahmen dieser Untersuchungen aufgestellten Versuchsreihen fiel zeitlich gesehen bereits in die Endphase der unter natürlichen Bedingungen ablaufenden Oocyten-Entwicklung. Alle 30 Versuchstiere (mittlere Segmentanzahl bei Versuchsbeginn: 193,7 bzw. 192,1), die sich im dementsprechend auf 31 Versuchstage verkürzten Experiment befanden, überlebten die gesamte Versuchsdauer. Auch in diesem weit fortgeschrittenen Stadium der Oocyten-Entwicklung tritt der aus den dreí vorangegangenen Versuchsreihen bereits gewohnte, wenn auch deutlich abgeschwächte Effekt eines beschleunigten OocytenWachstums nach Amputation des Prostomiums zu Tage (Abb. 7). 


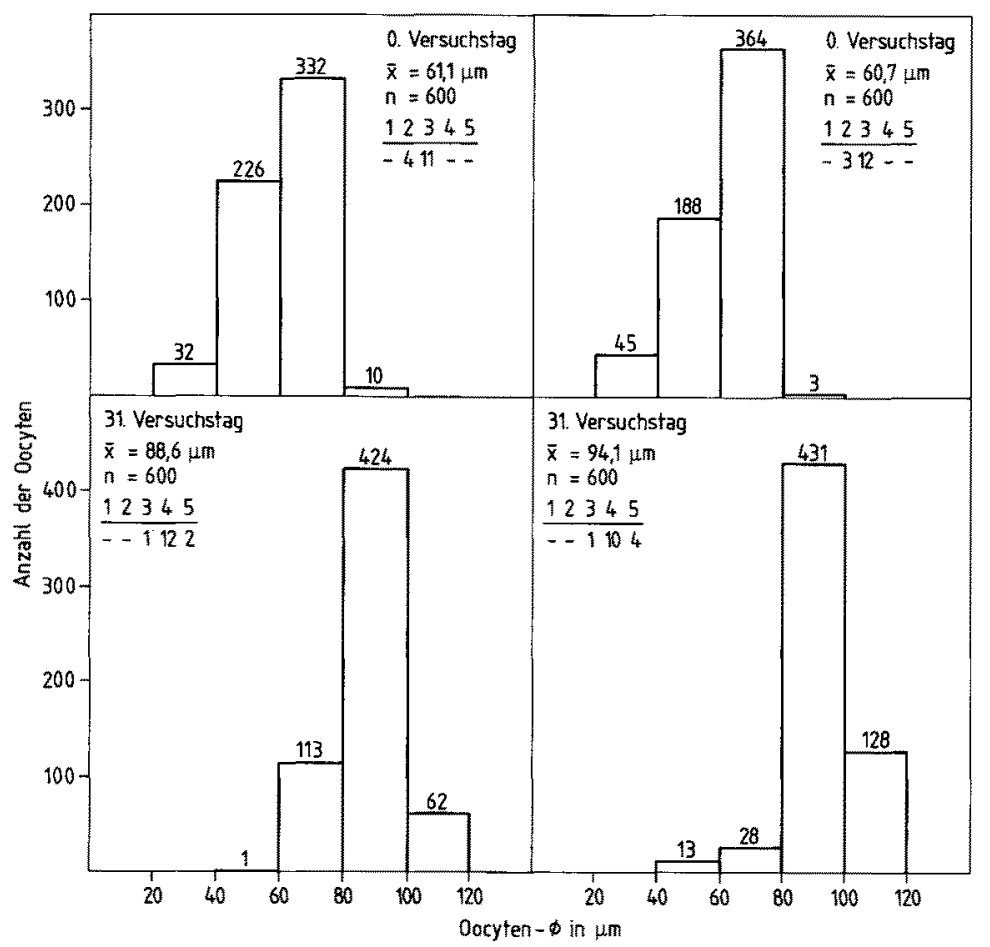

Abb. 7. Verteilung der Oocytengröße von Hungertieren (links) und dekapitierten Tieren (rechts) im Rahmen der Versuchsreihe 4

\section{Entwicklung der Oocyten nach Amputation und sofortiger Reimplantation des Prostomiums}

Um die Auswirkungen der Prostomium-Amputation auf die Oocyten-Entwicklung besser verstehen zu können, wurde im Rahmen der Versuchsreihe 2 eine dritte, aus 18 Tieren bestehende Versuchsgruppe (mittlere Segmentanzahl bei Versuchsbeginn: 210,0 ) eingerichtet. Sämtlichen Versuchstieren wurde das zuvor entfernte Prostomium durch eine seitliche Körperöffnung, durch die Abtrennung eines Parapodiums künstlich erzeugt, wieder ins Coelom gesteckt. Durch diese Maßnahme sollte die Gewähr für einen auch weiterhin ungehinderten Stoffaustausch zwischen dem Prostomium und den Segmenten gegeben sein. Bei einer hormonalen Grundlage der durch die Dekapitierung hervorgerufenen Vorgänge wäre demnach im günstigsten Fall eine den intakten Kontrolltieren entsprechende Oocyten-Entwicklung zu erwarten gewesen.

Ein Vergleich der Abb. 4 und 8 zeigt jedoch weitestgehende Ubereinstimmung dieser dritten Versuchsgruppe mit derjenigen der dekapitierten Versuchstiere. Ähnliche, wenn auch an dieser Stelle nicht näher ausgeführte Ergebnisse wurden darüber hinaus mit Versuchstieren erzielt, denen nicht nur das eigene zuvor amputierte Prostomium implantiert wurde, sondern zusätzlich noch zwei weitere von Tieren, die hinsichtlich ihrer Oocyten-Entwicklung derselben Klasse wie der jeweilige Empfänger angehörten. 


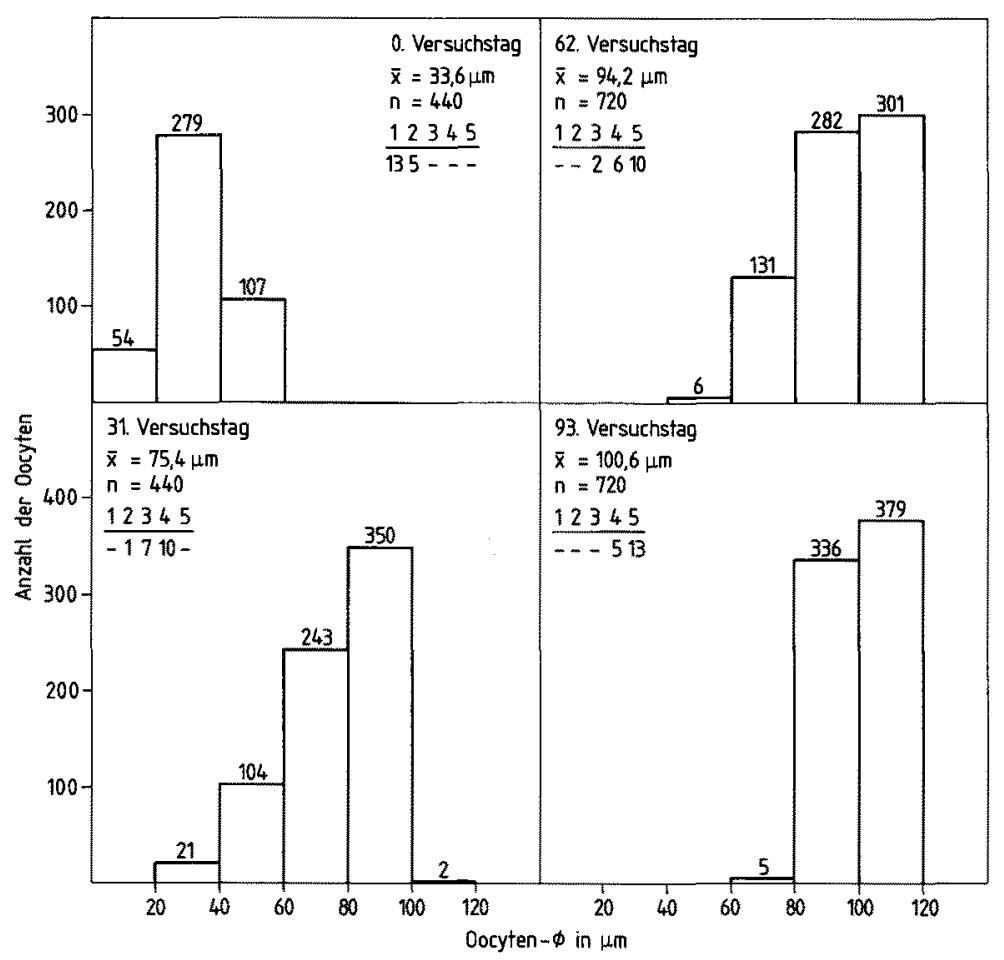

Abb. 8. Verteilung der Oocytengröße von Tieren nach Prostomium-Amputation mit sofort anschlieBender Reimplantation im Rahmen der Versuchsreihe 2

Da aus der Literatur für einige andere Familien der Polychaeten sowie für die Oligochaeten und Hirudineen ein Immunsystem bekannt ist, das für eine schnelle Zerstörung implantierter nervöser Strukturen verantwortlich ist und somit den experimentellen Nachweis hormonaler Aktivitäten beträchtlich erschwert, erfolgte eine histologische Untersuchung der entsprechenden Versuchstiere. Dabei zeigte es sich, daß die implantierten Prostomien auch noch drei Monate nach der Operation vollkommen intakt waren; ein ungehinderter Stoffaustausch mit der umgebenden Coelomflüssigkeit war ebenfalls gewährleistet. Auf Grund dieser Erkenntnisse muß angenommen werden, daß der hemmende Einfluß des Prostomiums über den Bauchganglienstrang wirksam wird. Ob es erst hier zur Ausschüttung von im Prostomium gebildeten Neurosekreten kommt oder ob nachgeschaltete Instanzen ihrerseits zur Bildung von Wirkstoffen, die die Oocyten-Entwicklung nachhaltig beeinflussen, veranlaßt werden, läßt sich beim gegenwärtigen Stand der Untersuchungen nicht abschätzen.

\section{DISKUSSION}

Seit den ersten Untersuchungen von Durchon (1952) und Hauenschild (1956) ist im Laufe der Jahre in zunehmendem Maße ein Bild von der Komplexität des neuroendokrinen Systems der Polychaeten entstanden. Die bisherigen Ergebnisse basieren dabei im 
wesentlichen auf histologischen Studien, die zur Entdeckung zahlreicher neurosekretorisch tätiger Zellen geführt haben, und auf physiologischen Arbeiten, insbesondere Ausschaltungs- und Transplantations-Experimenten. Bei fast allen in dieser Hinsicht experimentell untersuchten Arten erwies sich das Prostomium als Zentrum einer hormonalen Kontrolle der Fortpflanzungsvorgänge. Eine Ausnahme hiervon bilden lediglich die Sylliden. Bei den Angehörigen dieser Familie nimmt die Pharynxregion die entsprechenden Aufgaben wahr. Aber auch bei den Arten, deren Fortpflanzungsgeschehen der Steuerung durch neurosekretorische Zellen des Gehirns unterliegt, bestehen untereinander wiederum beträchtliche Unterschiede in der Art und Weise der Einflußnahme.

Die vorliegenden Untersuchungen an Anaitides mucosa zeigen mit großer Deutlichkeit die Abhängigkeit des zeitlichen Verlaufs der geschlechtlichen Entwicklung, sowohl bei den männlichen als auch unter den weiblichen Tieren, von einem im Prostomium lokalisierten, über den Bauchganglienstrang wirksam werdenden hemmenden Mechanismus. Insbesondere bei frühen Oogenese-Stadien ist die durch die Prostomium-Amputation auslösbare Beschleunigung der Oocyten-Entwicklung auffällig; die Beeinflußbarkeit späterer Stadien ist dagegen in zunehmendem Maße herabgesetzt. Der vom Prostomium ausgehende, die Entwicklung verlangsamende Einfluß dürfte somit im Hinblick auf den Beginn der Fortpflanzungssaison große Bedeutung erlangen. Inwieweit hierbei die reifenden Geschlechtsprodukte ihrerseits zum Erlöschen dieser hemmenden Wirkung beitragen, vermag zur Zeit noch nicht abgeschätzt zu werden.

Die zusammenfassende Darstellung der Ergebnisse aller vier Versuchsreihen in Tabelle 1 zeigt unter den intakten Kontrolltieren hinsichtlich des Zuwachses des mittleren Oocyten- $\varnothing$ während der ersten 31 Versuchstage große Übereinstimmung $(0,8-0,9 \mu \mathrm{m}$ pro Versuchstag). Dies steht durchaus im Einklang mit einer auch in gefütterten Massenkulturen weitestgehend linear erfolgenden Zunahme des Oocyten- $\varnothing$. Uber längere Versuchszeiträume betrachtet, erweist sich das Wachstum der Eizellen jedoch als abhängig vom Zeitpunkt des Versuchsbeginns, d. h. der Hungereffekt zeigt sich je deutlicher, desto geringere Werte der mittlere Oocyten- $\varnothing$ anfänglich besitzt.

Tab. 1. Zusammenfassung der Ergebnisse der Versuchsreihen 1-4 (Mittelwerte \pm mittlerer Fehler des Mittelwertes): $\mathrm{a}=$ intakte Kontrolltiere; $\mathrm{b}=$ prostomiumlose Tiere; $\mathrm{c}=$ dekapitierte Tiere mit reimplantiertem Prostomium

\begin{tabular}{|c|c|c|c|c|c|c|c|}
\hline \multirow[t]{2}{*}{$\begin{array}{l}\text { Versuchs- } \\
\text { reihe }\end{array}$} & \multicolumn{4}{|c|}{$\begin{array}{c}\text { Oocyten- } \varnothing(\mu \mathrm{m}) \\
\text { (Tag nach der Operation) }\end{array}$} & \multicolumn{3}{|c|}{$\begin{array}{c}\text { Zuwachs des Oocyten- } \varnothing \text { pro Tag }(\mu \mathrm{m}) \\
\text { (Zeitintervall) }\end{array}$} \\
\hline & 0. & 31 & 62. & 93 & $0-31$ & $31-62$ & $62-93$ \\
\hline $1 \mathrm{a}$ & - & $36,7 \pm 1,9$ & $48,1 \pm 2,5$ & $59,6 \pm 4,1$ & - & 0,37 & 0,37 \\
\hline $2 a$ & $35,0 \pm 1,8$ & $60,6 \pm 2,2$ & $78,7 \pm 3,0$ & $92,9 \pm 2,7$ & 0,83 & 0,58 & 0,46 \\
\hline $3 a$ & $45,8 \pm 1,8$ & $71,2 \pm 1,6$ & $95,3 \pm 1,4$ & - & 0,82 & 0,78 & - \\
\hline $4 \mathrm{a}$ & $61,1 \pm 2,1$ & $88,6 \pm 2,4$ & - & - & 0,89 & - & - \\
\hline $1 \mathrm{~b}$ & - & $52,9 \pm 3,7$ & $78,3 \pm 4,0$ & $87,6 \pm 3,7$ & - & 0,82 & 0,30 \\
\hline $2 b$ & $32,8 \pm 1,4$ & $77,2 \pm 2,4$ & $96,8 \pm 1,8$ & $100,5 \pm 0,8$ & 1,43 & 0,63 & 0,12 \\
\hline $3 b$ & $47,1 \pm 1,6$ & $79,8 \pm 2,1$ & $95,5 \pm 2,1$ & - & 1,05 & 0,51 & - \\
\hline $4 \mathrm{~b}$ & $60,7 \pm 1,6$ & $94,1 \pm 2,7$ & - & - & 1,08 & - & - \\
\hline $2 \mathrm{c}$ & $33,6 \pm 1,5$ & $75,4 \pm 3,4$ & $94,2 \pm 2,8$ & $100,6 \pm 1,4$ & 1,35 & 0,61 & 0,21 \\
\hline
\end{tabular}


Die Ergebnisse der Ausschaltungs- und Reimplantations-Experimente bei $A$. mucosa stehen offensichtlich im deutlichen Gegensatz zu denjenigen, die Olive (1975, 1976) durch entsprechende Untersuchungen an der nahe verwandten Phyllodocide Eulalia viridis erhielt. Bei dieser zuletzt genannten Art führt eine Prostomium-Amputation zur Degeneration der noch unreifen Oocyten, entsprechend ihrer Zugehörigkeit zur Gruppe der polytelischen Polychaeten mit gonadotrophen Hormonen. E. viridis besitzt gegenüber $A$. mucosa ferner die Fähigkeit zur vollständigen Regeneration des dekapierten Prostomiums, allerdings ohne dabei dessen inkretorische Aktivität wiederzuerlangen.

Allein diese Befunde an zwei Vertretern aus ein und derselben Familie geben somit eine Vorstellung von der Vielgestaltigkeit der innerhalb der Polychaeten verwirklichten Mechanismen zur Steuerung der Fortpflanzung und warnen zugleich vor übereilten Verallgemeinerungen.

Danksagungen. Für die Förderung dieser Arbeit danke ich Herrn Prof. Dr. C. Hauenschild, Zoologisches Institut der Universität Braunschweig. Herr Dr. H.-D. Franke, Zoologisches Institut der Universität Berlin, ermöglichte die Makrophotos.

\section{ZITIERTE LITERATUR}

Cazaux, C., 1969. Etude morphologique du développement larvaire d'annélides polychètes (Bassin d'Arcachon). II. Phyllodocidae, Syllidae, Nereidae. - Archs Zoll. exp. gén. 110, 145-202.

Durchon, M., 1952. Recherches expérimentales sur deux aspects de la reproduction chez les Annélides Polychètes: L'épitoquie et la stolonisation. - Annls Sci. nat. (Zool. Biol. anim.) 14, $117-206$.

Golding, D. W., 1974, A survey of neuroendocrine phenomena in non-arthropod invertebrates. Biol. Rev. 49, 161-224.

Gravier, C., 1896. Recherches sur les Phyllodociens. - Bull. scient. Fr. Belg. 29, 298-389.

Hauenschild, C., 1956. Hormonale Hemmung der Geschlechtsreife und Metamorphose bei dem Polychaeten Platynereis dumerilii. - Z. Naturforsch. 11b, 125-132.

Hauenschild, C., 1966. Der hormonale Einfluß des Gehirns auf die sexuelle Entwicklung bei dem Polychaeten Platynereis dumerilii. - Gen. comp. Endocrinol. 6, 26-73.

Hauenschild, C., 1974. Endokrine Beeinflussung der geschlechtlichen Entwicklung einiger Polychaeten. - Fortschr. Zool. 22 (2/3), 75-92.

Hauenschild, C., 1975. Die Beteiligung endokriner Mechanismen an der geschlechtlichen Entwicklung und Fortpflanzung von Polychaeten. - Verh. dt. zool. Ges. 67, 292-308.

Olive, P. J. W., 1975. A vitellogenesis promoting influence of the prostomium in the polychaete Eulalia viridis (Müller) (Phyllodocidae). - Gen. comp. Endocrinol. 26, 266-273.

Olive, P. J. W., 1976. Further evidence of a vitellogenesis promoting hormone and its activity in Eulalia viridis (Müller) (Polychaeta: Phyllodocidae). - Gen. comp. Endocrinol, 30, 397-403.

Olive, P. J. W., 1979. Endocrine adaptations in Annelida. In: Hormones and evolution. Ed. by E. J. M. Barrington. Acad. Press, New York, 73-118.

Olive, P. J. W. \& Clark, R. B., 1978. Physiology of reproduction. In: Physiology of annelids. Ed. by P. J. Mill. Acad. Press, London, 271-368.

Olive, P. J. W. \& Moore, F. R., 1975. Hormone independent regeneration in Eulalia viridis (Polychaeta: Phyllodocidae). - Gen. comp. Endocrinol. 28, 454-460.

Sach, G., 1975. Zur Fortpflanzung des Polychaeten Anaitides mucosa. - Mar. Biol. 31, 157-160. 